\title{
Chapter 34 \\ Involvement of Carbohydrate Residues \\ of the Zona Pellucida in In Vitro Sperm \\ Recognition in Pigs and Cattle
}

\author{
Naoto Yonezawa
}

\begin{abstract}
The zona pellucida (ZP), which surrounds the mammalian oocyte, plays roles in species-selective sperm-oocyte interactions. In pigs and cattle, the ZP consists of ZP2, ZP3, and ZP4. Nonreducing terminal $\beta$-galactosyl (Gal) residues of neutral $N$-linked carbohydrate chains of the ZP are necessary for porcine sperm-ZP binding, and nonreducing terminal $\alpha$-mannosyl (Man) residues of high-mannosetype chains are necessary for bovine sperm-ZP binding. Acrosome-intact porcine sperm prefer $\beta$-Gal, whereas acrosome-intact bovine sperm prefer $\alpha$-Man, as shown using glycolipid analogues. The major $N$-linked chains of recombinant porcine and bovine ZP glycoproteins expressed using the baculovirus-Sf9 cell expression system are pauci- and high-mannose-type chains that are different in structure from the major neutral $N$-linked chains of the native porcine ZP but similar to those of the native bovine ZP. Porcine and bovine ZP3/ZP4 complexes coexpressed in Sf9 cells bind to bovine sperm but not to porcine sperm. Hybrid complexes consisting of native porcine $\mathrm{ZP} 4$ and recombinant porcine $\mathrm{ZP} 3$ bind to porcine sperm, whereas complexes consisting of native porcine $\mathrm{ZP} 3$ and recombinant porcine $\mathrm{ZP} 4$ do not bind to porcine sperm. These data indicate that the sugar preference of sperm is consistent with the nonreducing terminal residues of $N$-linked chains of sperm binding-active ZP glycoproteins and suggest that, in the in vitro assay system, the nonreducing terminal sugar residues are essential for species-selective recognition of sperm in pigs and cattle.
\end{abstract}

Keywords Baculovirus-Sf9 cell $\bullet N$-linked chains $\bullet$ Sperm ligand $\bullet$ Zona pellucida

\author{
N. Yonezawa $(\triangle)$ \\ Graduate School of Science, Chiba University, 1-33 Yayoi-cho, \\ Inage-ku, Chiba 263-8522, Japan \\ e-mail: nyoneza@faculty.chiba-u.jp
}




\subsection{Introduction}

Mammalian oocytes are surrounded by a transparent envelope called the zona pellucida (ZP), which is involved in several critical aspects of fertilization, including species-selective recognition of sperm. In many mammalian species, there are four ZP glycoproteins (ZPGs) (ZP1, ZP2, ZP3, ZP4) (Stetson et al. 2012), although there are three ZPGs (ZP1, ZP2, ZP3) in mice. The porcine and bovine ZPs are composed of three ZPGs (ZP2, ZP3, ZP4). All ZPGs contain the ZP domain, which consists of about 260 amino acids and contains eight conserved Cys residues (Jovine et al. 2005).

Many studies have proposed that in mice the carbohydrate chains of ZP3 play an essential role in sperm recognition (the glycan model). However, a series of studies using transgenic mice do not support the glycan model (Hoodbhoy and Dean 2004). Instead, the supramolecular complex model, in which the supramolecular structure of the ZP polypeptides is necessary for sperm recognition in mice, was proposed based on studies using transgenic mice rescued by the human ZP2 gene (Rankin et al. 2003). Furthermore, the domain-specific model, in which both protein and carbohydrate moieties in the domain(s) of ZPGs are involved in sperm recognition, was recently proposed (Clark 2011). More recently still, it was shown using transgenic mice that sperm-ZP binding is not necessary for fertilization of oocytes surrounded by the cumulus oophorus (Tokuhiro et al. 2012). Whether this finding is applicable to mammals other than mice remains to be determined.

One of our research questions is whether carbohydrate chains are involved in sperm-ZP binding in pigs and cattle. Here, we discuss this subject mainly by reviewing our biochemical studies.

\subsection{Polypeptides of Porcine and Bovine ZPGs}

The porcine ZP is approximately $16 \mu \mathrm{m}$ in width and contains 30-33 ng glycoproteins. The bovine ZP is of similar size to the porcine ZP. The estimated ZP2/ZP3/ $\mathrm{ZP} 4$ protein molar ratio is $1: 6: 6$ in the porcine $\mathrm{ZP}$ and $1: 2: 1$ in the bovine $\mathrm{ZP}$. In mice, the estimated $\mathrm{ZP} 1 / \mathrm{ZP} 2 / \mathrm{ZP} 3$ molar ratio is $1: 4: 4$, and $\mathrm{ZP} 2$ and $\mathrm{ZP} 3$ form a filamentous equimolar complex, whereas ZP1 crosslinks the ZP2/ZP3 complex (Greve and Wassarman 1985). Based on the similarity in the protein molar ratios, it appears that $\mathrm{ZP}$ architecture is similar in pigs and mice but bovine $\mathrm{ZP}$ architecture appears to differ from that of porcine and murine $\mathrm{ZP}$.

Cys positions of the ZP domain are classified into two patterns: the ZP3 pattern and the ZP1/ZP2/ZP4 pattern (Fig. 34.1). Porcine ZP3 and ZP4 exhibit disulfide patterns different from the $\mathrm{ZP} 3$ and $\mathrm{ZP} 1 / \mathrm{ZP} 2 / \mathrm{ZP} 4$ patterns reported for mice, rats, humans, and fish (Kanai et al. 2008). The chick homologue of the mammalian ZP3 precursor protein has a pig-type ZP3 pattern (Han et al. 2010), whereas betaglycan has a pig-type ZP1/ZP2/ZP4 pattern (Lin et al. 2011), as revealed by X-ray crystallography. The two ZP3 disulfide bond patterns cause only subtle structural differences (Han et al. 2010). The subtle structural differences may affect the specificity of 


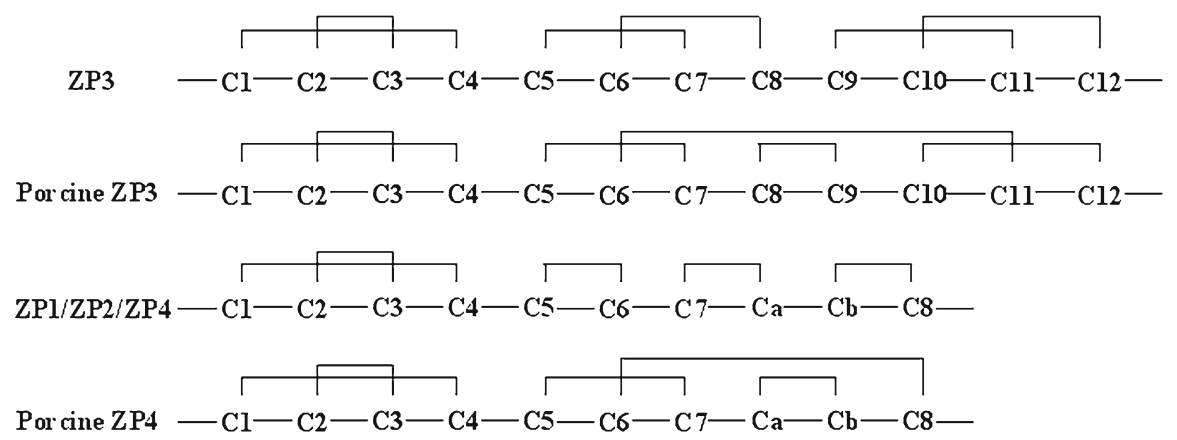

Fig. 34.1 Schematic representations of disulfide bond patterns in zona pellucida (ZP) 3 and ZP1/ $\mathrm{ZP}$ /ZP4. The disulfide bond pattern of ZP3 from mice, human, rat and fish, the pattern of porcine $\mathrm{ZP} 3$, the pattern in the ZP domain of ZP1/ZP2/ZP4-type proteins from mice, rat and fish, the pattern in the ZP domain of porcine ZP4 are shown schematically. The patterns of $\mathrm{C} 1-\mathrm{C} 4$ are completely conserved in the ZP domain proteins. $C$ Cys

the interaction between ZP1/ZP2/ZP4 and ZP3 (Darie et al. 2004). When the disulfide bond pattern of $\mathrm{ZP} 3$ is of the pig type, the $\mathrm{ZP} 1 / \mathrm{ZP} 2 / \mathrm{ZP} 4$ pattern of the same species is also the pig type, and vice versa. The disulfide bond patterns might change during zona matrix formation, following processing at the consensus furin cleavage site.

\subsection{N-Linked Carbohydrate Chains from Porcine and Bovine ZPGs}

The $N$-linked chains of porcine ZP comprise neutral and acidic chains at a molar ratio of about 1:3. Both neutral chains and acidic chains comprise di-, tri-, and tetraantennary complex-type chains with an $\alpha$-fucosyl (Fuc) residue at the innermost $N$-acetylglucosamine (GlcNAc) (Nakano and Yonezawa 2001). A large proportion of neutral chains have nonreducing terminal $\beta$-galactosyl (Gal) residues.

In the bovine $\mathrm{ZP}$, the major neutral $N$-linked chain consists of only one structure: the high-mannose-type chain containing five mannosyl (Man) residues $\left(\mathrm{Man}_{5} \mathrm{GlcNAc}_{2}\right.$ ) (Katsumata et al. 1996). Sialylation is predominant in acidic chains of bovine $\mathrm{ZP}$; in porcine $\mathrm{ZP}$, sulfation of the $\mathrm{N}$-acetyl lactosamine unit is more dominant.

\subsection{Involvement of Carbohydrate Chains from Porcine and Bovine ZPGs in Sperm-ZP Binding}

Neutral $N$-linked carbohydrate chains released from the porcine ZP3/ZP4 mixture retain their sperm-binding activities, with the tri- and tetra-antennary complextype chains binding more strongly than the di-antennary complex-type chains 
(Nakano and Yonezawa 2001). In one study, the nonreducing terminal $\beta$-Gal residues of the complex-type $N$-linked chains were shown to be involved in sperm binding (Yonezawa et al. 2005a). Conversely, another study reported that the $O$-linked carbohydrate chains, and not the $N$-linked chains, released from the ZP3/ ZP4 mixture inhibit sperm-ZP binding (Yurewicz et al. 1991). Therefore, both the $\mathrm{N}$ - and $\mathrm{O}$-linked carbohydrate chains are thought to be sperm ligands. A polypeptide scaffold is necessary for the sperm-binding activity of carbohydrate chains, because the sperm-binding activities of carbohydrate chains released from polypeptides are much weaker than the activity of the ZP3/ZP4 mixture.

Porcine ZP3 and ZP4 have three $N$-linked chains, at Asn124, Asn146, and Asn271 in ZP3 and at Asn203, Asn220, and Asn333 in ZP4. Tri- and tetra-antennary chains are localized at Asn271 in ZP3 and at Asn220 in ZP4 (Nakano and Yonezawa 2001). ZP2 has five $N$-glycosylation sites: Asn84, Asn268, Asn316, Asn323, and Asn530 (von Witzendorff et al. 2005). A remarkable difference between the $N$-linked chain structures of $\mathrm{ZP} 2$ and ZP3/ZP4 is that ZP2 has a high-mannose-type chain containing five Man residues, probably located at Asn268.

Nonreducing terminal $\alpha$-Man residues of the high-mannose-type chain $\mathrm{Man}_{5} \mathrm{GlcNAc}_{2}$ play an essential role in bovine sperm-ZP binding (Amari et al. 2001). Sialic acid residues at the nonreducing ends of acidic $N$-linked or $O$-linked chains of bovine ZPGs are also involved in sperm binding (Velásquez et al. 2007). The $N$-glycosylation sites of bovine ZP3 and ZP4 have not yet been determined. The $N$-glycosylation sites of bovine ZP2 are Asn83, Asn191, and Asn527 (Ikeda et al. 2002).

Glycolipid analogue-possessing monosaccharides adsorbed on plastic wells show sperm-binding activity, but monosaccharide solution does not inhibit spermZP binding (Takahashi et al. 2013). Two-dimensional coating of plastic wells with glycolipid analogue may cause the formation of a cluster of sugars, which is necessary for the high avidity for sperm binding. Acrosome-intact porcine sperm exhibit their strongest affinity for plastic wells coated with $\beta$-Gal and the second strongest affinity for $\beta$ - $N$-acetylgalactosamine (GalNAc), whereas acrosome-intact bovine sperm exhibit their strongest binding affinities for $\alpha$-Man and their second strongest affinity for $\beta$-glucose (Glc) and $\beta$-GlcNAc. These specificities are consistent with the nonreducing terminal sperm ligand sugar residues of porcine and bovine ZPs.

\subsection{Sperm Binding-Active Regions of Porcine and Bovine ZPGs}

We identified the sperm binding-active fragment of porcine ZP4 as an N-terminal region (Asp137 to Lys247) containing two $N$-linked chains (Nakano and Yonezawa 2001). Porcine ZP4 purified by reverse-phase HPLC is actually contaminated with $\mathrm{ZP} 3$, and formation of a heterocomplex of ZP3 and ZP4 is essential for the spermbinding activity of the glycoproteins (Yurewicz et al. 1998). We established an expression system for recombinant porcine ZPGs (rpZPGs) using baculovirus-Sf9 


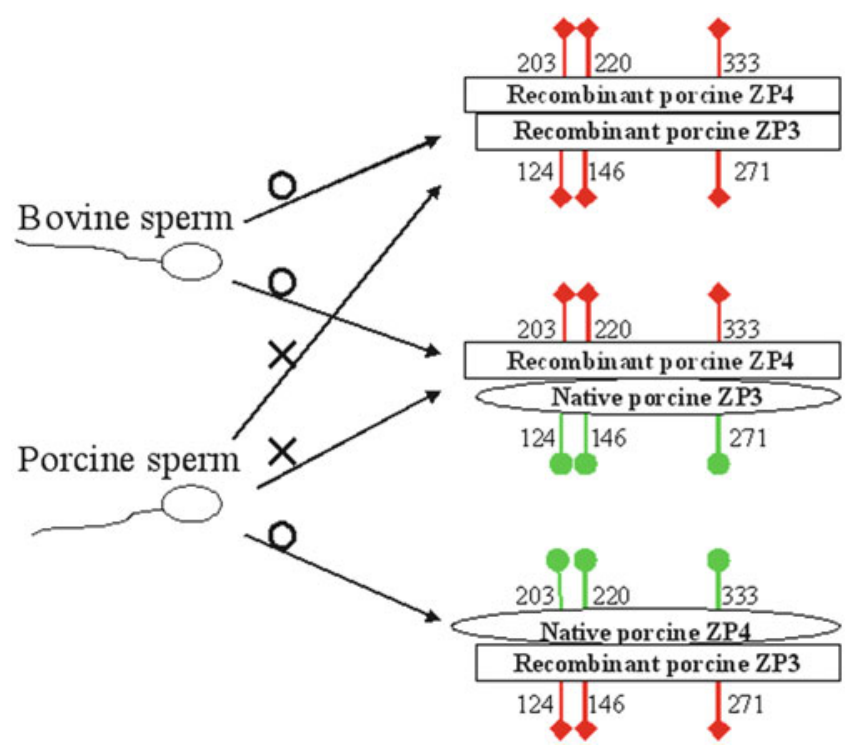

Fig. 34.2 Schematic representation of the specificity in recognition between sperm and zona pellucida (ZP) glycoproteins. Porcine sperm bind to the heterocomplex of porcine ZP3 and ZP4 in which ZP4 has complex-type $N$-linked chains (closed circles in green) but not to the heterocomplexes in which ZP4 has pauci- and high-mannose-type chains (closed diamonds in red). On the other hand, bovine sperm bind to the heterocomplexes in which ZP4 has pauci- and high-mannosetype chains

cells to obtain ZP4 without contamination of ZP3 (Yonezawa et al. 2005b). The major structures of the $N$-linked chains of rpZPGs are believed to be pauci- and high-mannose-type chains with or without a Fuc residue at the innermost GlcNAc. They have nonreducing terminal $\alpha$-Man residues and are thus similar in structure to the sperm ligand $N$-linked chain of bovine ZPGs. The rpZP3/rpZP4 mixture coexpressed by Sf9 cells shows binding activity toward bovine sperm but not to porcine sperm (Fig. 34.2). The sperm-binding activity of rpZP4 is much weaker than that of rpZP3/rpZP4 (Yonezawa et al. 2005b). This study supports the previous report by Yurewicz et al. (1998).

To reexamine the sperm binding-active region of porcine ZP4, rpZP4 mutants with an Asp substitution at Asn203, Asn220, or Asn333 were coexpressed with rpZP3 in Sf9 cells (Yonezawa et al. 2005b). The mutation at Asn333 does not have an effect on the sperm-binding activity of the rpZP3/rpZP4 mixture, whereas the mutations at Asn203 and Asn220 reduce the sperm-binding activity secondarily and primarily among the three sites, respectively. These results are in agreement with our previous finding that the $\mathrm{N}$-terminal fragment containing two $\mathrm{N}$-linked chains at Asn203 and Asn220 exhibits sperm-binding activity, and that the tri- and tetraantennary chains are localized at Asn220 in porcine ZP4.

Porcine sperm bind to native porcine ZPGs, but not to rpZPGs, probably because of differences in carbohydrate structures between native and recombinant ZPGs. A mixture of rpZP3 and native porcine ZP4 binds to the acrosomal region of porcine 
sperm and inhibits porcine sperm-ZP binding (Fig. 34.2) (Yonezawa et al. 2012). A mixture of native porcine $\mathrm{ZP} 3$ and rpZP4 does not inhibit the binding, although the mixture inhibits bovine sperm-ZP binding. This study indicated that native ZP4, but not $\mathrm{rZP} 4$, is necessary for the binding activity of the porcine ZP3/ZP4 complex toward porcine sperm and further suggested that the carbohydrate structures of ZP4 in the porcine $\mathrm{ZP} 3 / \mathrm{ZP} 4$ complex are responsible for the porcine sperm-binding activity of the complex. Thus, in the present model for porcine sperm-ZP binding, the $N$-linked chains with nonreducing terminal $\beta$-Gal residues linked to the $\mathrm{N}$-terminal region of $\mathrm{ZP} 4$ form a sperm-binding domain in the ZP3/ZP4 heterocomplex.

ZP4 has the highest sperm-binding activity among bovine ZPGs purified from ovaries, while ZP2 and ZP3 have weak but significant sperm-binding activities (Yonezawa et al. 2001). In the case of bovine ZPGs, ZP4 is not completely purified by reverse-phase HPLC and is contaminated with ZP3. To obtain ZP3 and ZP4 without contamination of other ZPGs, we established an expression system for bovine ZPGs using the baculovirus-Sf9 cell system (Kanai et al. 2007). Recombinant bovine ZP3 (rbZP3) and rbZP4 do not show sperm-binding activity, whereas the rbZP3/ rbZP4 complex does show sperm-binding activity. The region from the $\mathrm{N}$-terminus to the trefoil domain of rbZP4 is dispensable for formation of the complex with rbZP3 and for the sperm-binding activity of the complex. More detailed analysis of the sperm-binding region of bovine ZP3/ZP4 has not been accomplished.

\subsection{Conclusion}

Our studies indicate that porcine and bovine sperm proteins recognize $\beta$-Gal and $\alpha$-Man residues, respectively, at the nonreducing ends of carbohydrate chains linked to the ZP3/ZP4 complex. In pigs and cattle, ZP3 and ZP4 form a framework on which carbohydrate chains active in sperm binding can exhibit high avidity for sperm. To prove the significance of the carbohydrate chains in sperm binding, the sperm proteins that bind specifically to $\beta$-Gal in pigs and $\alpha$-Man in cattle need to be identified and characterized.

Open Access: This article is distributed under the terms of the Creative Commons Attribution Noncommercial License which permits any noncommercial use, distribution, and reproduction in any medium, provided the original author(s) and source are credited.

\section{References}

Amari S, Yonezawa N, Mitsui S et al (2001) Essential role of the nonreducing terminal $\alpha$-mannosyl residues of the $N$-linked carbohydrate chain of bovine zona pellucida glycoproteins in spermegg binding. Mol Reprod Dev 59:221-226

Clark GF (2011) Molecular models for mouse sperm-oocyte binding. Glycobiology 21:3-5

Darie CC, Biniossek ML, Jovine L et al (2004) Structural characterization of fish egg vitelline envelope proteins by mass spectrometry. Biochemistry 43:7459-7478 
Greve JM, Wassarman PM (1985) Mouse egg extracellular coat is a matrix of interconnected filaments possessing a structural repeat. J Mol Biol 181:253-264

Han L, Monné M, Okumura H et al (2010) Insights into egg coat assembly and egg-sperm interaction from the X-ray structure of full-length ZP3. Cell 143:404-415

Hoodbhoy T, Dean J (2004) Insights into the molecular basis of sperm-egg recognition in mammals. Reproduction 127:417-422

Ikeda K, Yonezawa N, Naoi K et al (2002) Localization of $N$-linked carbohydrate chains in glycoprotein ZPA of the bovine egg zona pellucida. Eur J Biochem 269:4257-4266

Jovine L, Darie CC, Litscher ES et al (2005) Zona pellucida domain proteins. Annu Rev Biochem 74:83-114

Kanai S, Yonezawa N, Ishii Y et al (2007) Recombinant bovine zona pellucida glycoproteins ZP3 and ZP4 coexpressed in Sf9 cells form a sperm-binding active hetero-complex. FEBS J 274:5390-5405

Kanai S, Kitayama T, Yonezawa N et al (2008) Disulfide linkage patterns of pig zona pellucida glycoproteins ZP3 and ZP4. Mol Reprod Dev 75:847-856

Katsumata T, Noguchi S, Yonezawa N et al (1996) Structural characterization of the $N$-linked carbohydrate chains of the zona pellucida glycoproteins from bovine ovarian and fertilized eggs. Eur J Biochem 240:448-453

Lin SJ, Hu Y, Zhu J et al (2011) Structure of betaglycan zona pellucida (ZP)-C domain provides insights into ZP-mediated protein polymerization and TGF-beta binding. Proc Natl Acad Sci USA 108:5232-5236

Nakano M, Yonezawa N (2001) Localization of sperm ligand carbohydrate chains in pig zona pellucida glycoproteins. Cells Tissues Organs 168:65-75

Rankin TL, Coleman JS, Epifano O et al (2003) Fertility and taxon-specific sperm binding persist after replacement of mouse sperm receptors with human homologs. Dev Cell 5:33-43

Stetson I, Izquierdo-Rico MJ, Moros C et al (2012) Rabbit zona pellucida composition: a molecular, proteomic and phylogenetic approach. J Proteomics 75:5920-5935

Takahashi K, Kikuchi K, Uchida Y et al (2013) Binding of sperm to the zona pellucida mediated by sperm carbohydrate-binding proteins is not species-specific in vitro between pigs and cattle. Biomolecules 3:85-107

Tokuhiro K, Ikawa M, Benham AM et al (2012) Protein disulfide isomerase homolog PDILT is required for quality control of sperm membrane protein ADAM3 and male fertility. Proc Natl Acad Sci USA 109:3850-3855

Velásquez JG, Canovas S, Barajas P et al (2007) Role of sialic acid in bovine sperm-zona pellucida binding. Mol Reprod Dev 74:617-628

von Witzendorff D, Ekhlasi-Hundrieser M, Dostalova Z et al (2005) Analysis of $N$-linked glycans of porcine zona pellucida glycoprotein ZPA by MALDI-TOF MS: a contribution to understanding zona pellucida structure. Glycobiology 15:475-488

Yonezawa N, Fukui N, Kuno M et al (2001) Molecular cloning of bovine zona pellucida glycoproteins ZPA and ZPB and analysis for sperm-binding component of the zona. Eur J Biochem 268:3587-3594

Yonezawa N, Amari S, Takahashi K et al (2005a) Participation of the nonreducing terminal betagalactosyl residues of the neutral $N$-linked carbohydrate chains of porcine zona pellucida glycoproteins in sperm-egg binding. Mol Reprod Dev 70:222-227

Yonezawa N, Kudo K, Terauchi H et al (2005b) Recombinant porcine zona pellucida glycoproteins expressed in Sf9 cells bind to bovine sperm but not to porcine sperm. J Biol Chem 280: 20189-20196

Yonezawa N, Kanai-Kitayama S, Kitayama T et al (2012) Porcine zona pellucida glycoprotein ZP4 is responsible for the sperm-binding activity of the ZP3/ZP4 complex. Zygote 20:389-397

Yurewicz EC, Pack BA, Sacco AG (1991) Isolation, composition, and biological activity of sugar chains of porcine oocyte zona pellucida 55K glycoproteins. Mol Reprod Dev 30:126-134

Yurewicz EC, Sacco AG, Gupta SK et al (1998) Hetero-oligomerization-dependent binding of pig oocyte zona pellucida glycoproteins ZPB and ZPC to boar sperm membrane vesicles. J Biol Chem 273:7488-7494 